\title{
Another single hydrogen-rich Wolf-Rayet star in the SMC?^
}

\author{
C. Foellmi ${ }^{\star \star}$ \\ European Southern Observatory, Alonso de Cordova 3107, Vitacura, casilla 19001, Santiago, Chile \\ Received 6 October 2003 / Accepted 24 November 2003

\begin{abstract}
A 12th Wolf-Rayet star in the SMC has recently been discovered by Massey et al. (2003). To determine its spectral type and preliminary binary status, we obtained 3 high signal-to-noise spectra separated in time at the ESO-NTT. Compared to other WR stars in the SMC, SMC-WR12 appears to belong to the subgroup of faint, single and hydrogen-rich WN stars. We discuss the evolutionary status of WR12 and show that relatively low mass rotating progenitors can better account for the
\end{abstract} \\ properties of single hydrogen-rich WN stars in the SMC.
}

Key words. stars: Wolf-Rayet - stars: individual: SMC-WR12 - stars: rotation - galaxies: individual: SMC

\section{Introduction}

Until recently, the population of Wolf-Rayet (WR) stars in the SMC was considered nearly complete (see the discussion in Massey \& Duffy 2001). However, Massey et al. (2003) have discovered a 12th WR star in the SMC from the same survey as Massey \& Duffy (2001). The discovery was delayed because of a misidentification of the target during the spectroscopic confirmation. According to their discovery data, it has an early spectral type in the nitrogen sequence (WN3-4.5) with a $V$ magnitude of about 15.5. Unfortunately, these data do not allow a more precise determination of the evolutionary status of the star.

The discovery of another WR star in the SMC is important not only for the completeness issue, but also because the very low metallicity of the SMC makes it a very good laboratory to test the influence of metallicity on the evolution of WR stars, and the problem of their formation. This has a direct impact on studies of starbursts at low metallicity that use the SMC as a prototype (e.g. Schaerer \& Vacca 1998).

Recently, the 10 previously known WN stars in the SMC have been studied by Foellmi et al. (2003a). These authors provided new and consistent spectral types, based on homogeneous high- $S / N$ spectra. They have shown that a significant fraction, if not all WN stars have hydrogen absorption lines in their spectra and that these lines are clearly blue-shifted. They argued that hydrogen must be part of the WR, even for hot, early-type single WN stars. However, in their description of SMC-WR12, Massey et al. (2003) argue that "the presence of absorption spectra in the SMC WRs is still not well understood".

\footnotetext{
* Based on observations made at the European Southern Observatory, La Silla, Chile.

$\star \star$ e-mail: cfoellmi@eso.org
}

To complete the study of Foellmi et al. (2003a) and to discuss the evolutionary status of this new interesting object, we have obtained a set of spectroscopic observations of SMC-WR12. This allows us to make a rapid check of radialvelocity $(R V \mathrm{~s})$ variations, to measure the $R V \mathrm{~s}$ of the absorption lines present in the spectrum and to provide a reliable spectral type. In Sect. 2 we describe the observations and the $R V$ measurements, while Sect. 3 contains the discussion. Section 4 summarizes our conclusions.

\section{Observations}

We observed SMC-WR12 at the ESO-NTT during the nights of September 19, 20 and 29, 2003 (see the journal of observations in Table 1). The reader is referred to the paper of Massey et al. (2003) for identification, magnitude, coordinates and finding chart. We used the spectrograph EMMI on the Red side, with Grism\#5 and a slit of $1^{\prime \prime}$. This provides a resolution (FWHM) of $\sim 4.5 \AA$. This wavelength range covers the most important emission and absorption lines, from 4000 to $6800 \AA$. To calibrate the spectra for radial-velocities $(R V \mathrm{~s})$, an arc spectrum was taken before and after the science exposures. Unfortunately, due to technical problems with the HeAr calibration lamp in the EMMI spectrograph during the third night, the only calibration spectrum we have for this night is less reliable. A rectified $S / N$-weighted mean spectrum of SMC-WR12 is shown in Fig. 1.

To determine the spectral type we followed the classification scheme of Smith et al. (1996). In our spectrum, He I $\lambda 5875$ is absent. Although not a defining criterion, this points towards a very early ionization subclass (WN2-3), as does the absence of N III $\lambda \lambda 4634,4642$, N IV $\lambda 4058$ and C IV $\lambda 5808$. However $\mathrm{N} v \lambda \lambda 4603,4619$ is clearly visible, definitely making a WN3 ionization subclass for SMC-WR12. 


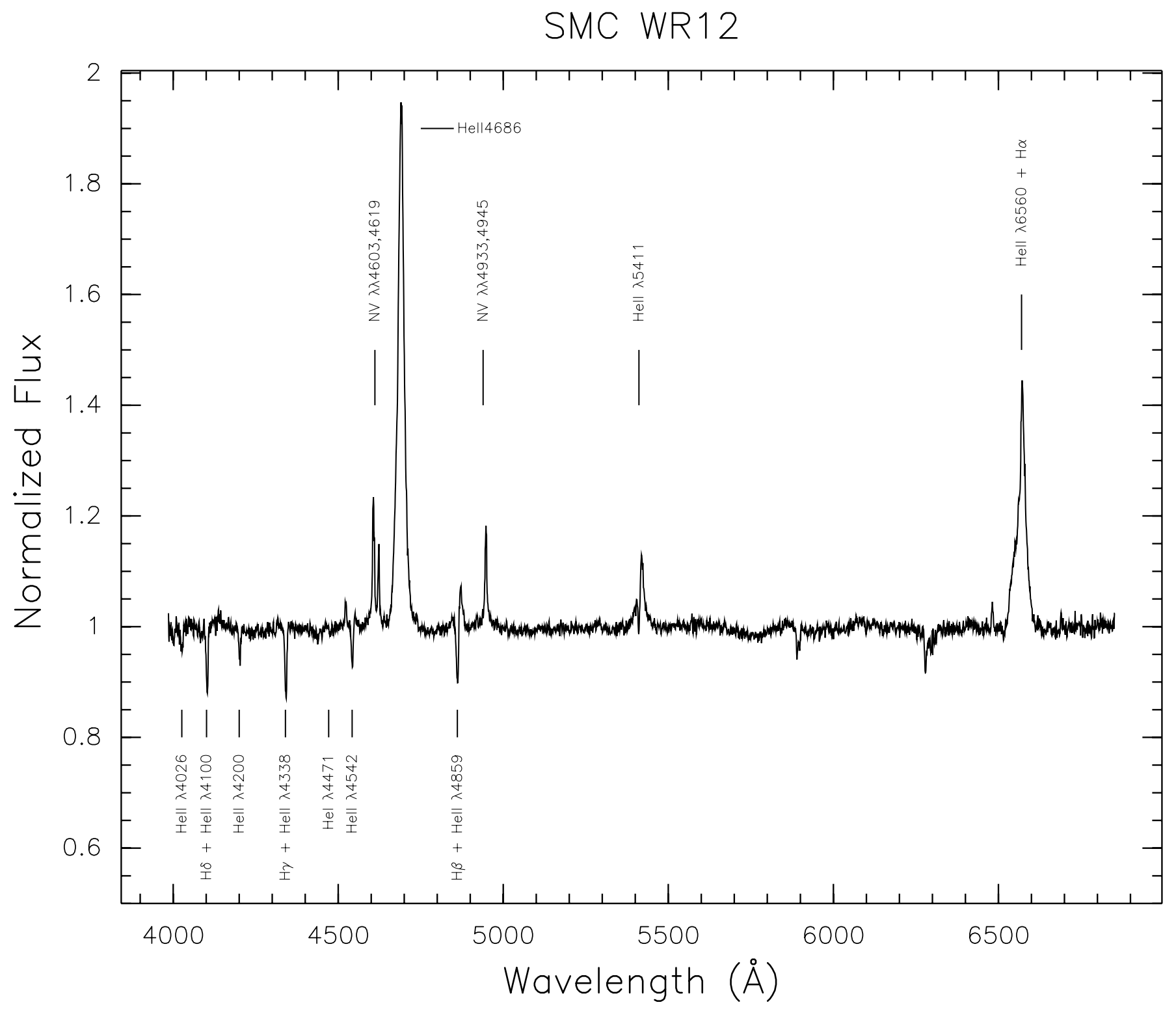

Fig. 1. Spectrum of SMC-WR12, obtained from a $S / N$-weighted mean of all spectra. We easily see hydrogen and helium absorption lines.

The time elapsed between the first and the last spectrum (10 days) allows us to perform a preliminary check for $R V$ variations of WR12. For that purpose, we also observed a bright star (namely $\zeta$ Dor $=$ HD 33262, spectral type F7V) with a known constant velocity to play the role of a $R V$ standard.

The spectra were first corrected to the heliocentric rest frame. Then, the $R V \mathrm{~s}$ of the 2 stars were measured with a crosscorrelation (CC) technique and bisectors. The procedure used is identical to the one described in Foellmi et al. (2003a), and the wavelength window for CC ranges from 4570 to $4800 \AA$ to avoid any possible influence from the absorption lines. The $R V \mathrm{~s}$ of SMC-WR12 and $\zeta$ Dor are plotted in Fig. 2. The figure shows that the spectrum of the third night is red-shifted for both stars, by a similar amount $\left(\sim 70 \mathrm{~km} \mathrm{~s}^{-1}\right)$.

Although we cannot rule out the possibility, with only 3 spectra over 10 days, that SMC-WR 12 could be a binary star with a low-inclination angle, the $R V$ s presented here are consistent with a single star (after correction of the third night).
Table 1. Journal of observations. The heliocentric Julian Date, the signal-to-noise ratio in the continuum and the total exposure time (in minutes) are indicated.

\begin{tabular}{lrc}
\hline \hline HJD & $S / N$ & total exp. \\
\hline 2452902.8243 & 86 & 85 \\
2452903.7696 & 99 & 60 \\
2452912.5273 & 121 & 120 \\
\hline
\end{tabular}

Additional data will obviously be needed to confirm this preliminary result.

To add weight to this presumed single star status, we have measured the $R V$ s of the absorption lines, using a Gaussian profile, on the (heliocentric) spectrum of each night. The central wavelengths of the lines were compared to their rest wavelength. The results are summarized in Table 2. 


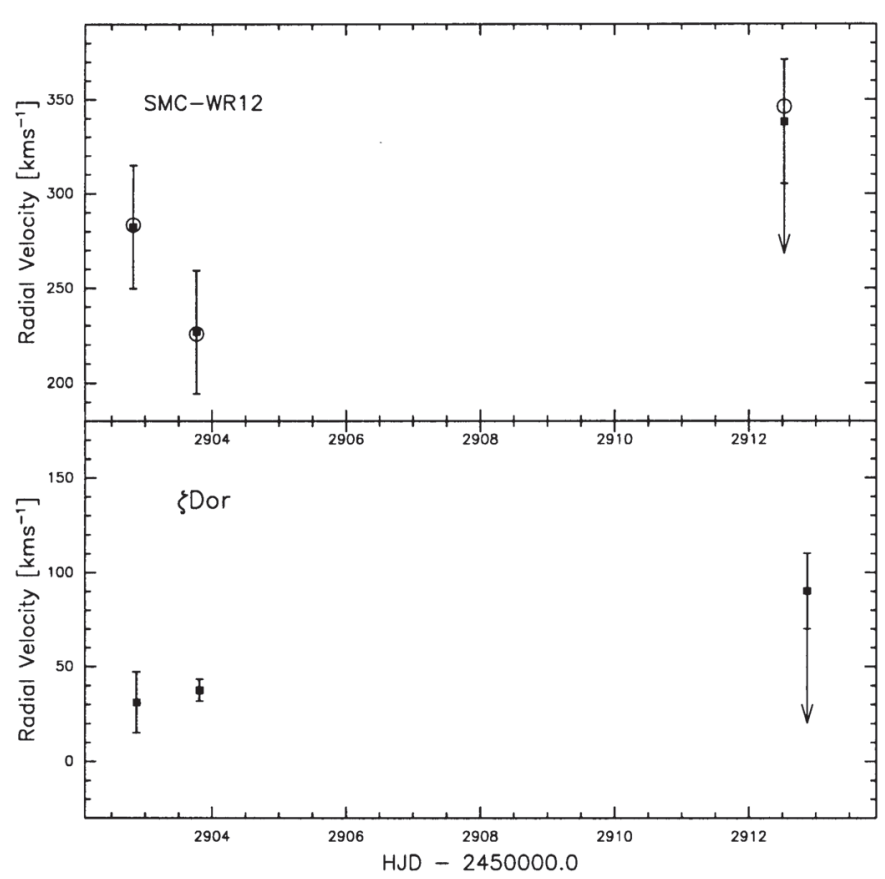

Fig. 2. Radial-velocities of SMC-WR12 (top), and $\zeta$ Dor (bottom). Both panels have the same vertical scale to allow comparison. For SMC-WR12, the $R V$ s have been measured with the cross-correlation method (filled squares) and the bissector method (open circles). For $\zeta$ Dor, only cross-correlation has been used. The red-shifted calibration solution of the third night is easily visible for both stars (the arrow indicates $-70 \mathrm{~km} \mathrm{~s}^{-1}$ ). The $R V \mathrm{~s}$ are consistant with a constant velocity (see text).

Similar to what is seen in other single WN stars in the SMC, these lines have a constant $R V$ and are clearly blue-shifted relative to the frame of the star, whose mean measured $R V$ (from the CC, see above) is $\sim 260 \mathrm{~km} \mathrm{~s}^{-1}$. However, the emission lines of WR stars are known to not reflect the true systemic velocity of the star, since the line profiles are often not symmetric, and might be distorted by the presence of an even weak P Cygni profile. Nevertheless, when compared to the mean $R V$ of the SMC $\left(160 \mathrm{~km} \mathrm{~s}^{-1}\right)$, the blue shift observed in the $R V \mathrm{~s}$ of the absorption lines of WR12 remains significant.

Note that for the blended lines, the resolution of the spectra does not allow a precise fitting by a double Gaussian (the resolution is $\sim 300 \mathrm{~km} \mathrm{~s}^{-1}$ while the lines are separated by $\sim 123 \mathrm{~km} \mathrm{~s}^{-1}$ ). The measured central wavelength has been compared to the simple mean of the rest wavelengths of the two lines. If the hydrogen line is dominant in the blend, the radial velocity measurement quoted in Table 2 is an upper limit. This makes the blueshift even stronger. If, on other hand, the helium line is dominant, a shift of $123 / 2=62 \mathrm{~km} \mathrm{~s}^{-1}$ needs to be added, and this would make the blueshift less obvious. However, the hydrogen influence must be nonetheless significant since blended lines have larger equivalent widths.

Therefore, the blueshift is real and the absorption lines of SMC-WR12 must be formed deep in the relatively weak WR wind, at modestly blue-shifted $R V$ along the line of sight to the stellar core. They do belong to the WR object itself, and cannot come from a binary companion (that would also contradict the combined observed constancy of the $R V \mathrm{~s}$ ).
Table 2. Radial-velocities and equivalent widths of absorption lines of SMC-WR12. The rest wavelength used for each (combination of) lines (Phelps 1982) is also indicated. For the combination of 2 lines, the mean wavelength has been computed. The $R V$ is the mean of $R V \mathrm{~s}$ measured on the 3 spectra. The estimated accuracy is $\sim 30 \mathrm{~km} \mathrm{~s}^{-1}$ on individual measurement. The accuracy has been estimated by making the Gaussian fit with different fraction of the line and/or the continuum. The variance of these various results give an estimation of the accuracy. Moreover, the accuracy can easily reach a fraction of pixel, usually a tenth of the resolution. With a $300 \mathrm{~km} \mathrm{~s}^{-1}$ resolution, the accuracy is about $30 \mathrm{~km} \mathrm{~s}^{-1}$.

\begin{tabular}{lrrr}
\hline \hline Line & $\lambda_{0}(\AA)$ & $R V\left(\mathrm{~km} \mathrm{~s}^{-1}\right)$ & $E W(\AA)$ \\
\hline H $\delta \lambda 4101+$ He II $\lambda 4100$ & 4100.87 & 58 & 0.76 \\
He II $\lambda 4200$ & 4199.85 & 35 & 0.40 \\
H $\gamma \lambda 4340+$ He II $\lambda 4338$ & 4339.58 & 53 & 0.94 \\
He II $\lambda 4542$ & 4541.66 & -9 & 0.57 \\
H $\beta \lambda 4861+$ He II $\lambda 4859$ & 4860.33 & -34 & 1.17 \\
\hline
\end{tabular}

Moreover, as argued in Foellmi et al. (2003a), the SMC WR stars whose emission lines do not satisfy the broadline criterium in the classification scheme of Smith et al. (1996) contain hydrogen (see Smith \& Maeder 1998). Additionaly, the presence of hydrogen can be assessed from the Balmer versus Pickering decrement. As seen in Table 2, the equivalent widths of the blended $\mathrm{H}+\mathrm{He}$ lines are larger than that of pure He lines, which indicates the presence of hydrogen in the spectrum (see also Hamann et al. 1991, for a detailed discussion). Although being defined for emission lines, the second criterion for the hydrogen in the Smith et al. classification (see their Table 4c) can be computed since the three relevant lines are all in absorption. The value obtained is 1.9 for SMC-WR12, making a strong case in favour of an "h" suffix.

Finally, the complete spectral type of WR12 is WN3ha. This spectral type makes even stronger the need for a complementary classification, as proposed by Foellmi et al. (2003b), since the star has a very early ionization subclass and a significant hydrogen content at the same time, the latter being more related to late-type $\mathrm{WN}$ stars. In that context, the evolutionary classification of SMC-WR12 is eWNL.

\section{Discussion}

This 12th WR star in the SMC, if proved to be single as seems to be the case, would lower even more the binary frequency of the SMC ( 40\%) found by Foellmi et al. (2003a). However, with only 12 stars, it remains small number statistics. Nevertheless, the consequence of the existence of this new member is that nearly half of the WR population is hydrogenrich with an early spectral type.

\subsection{Response to the conclusions of Massey et al.}

Massey et al. (2003) argue that the origin of absorption lines in WR spectra is either due to the fact that the WR winds are weak and we see photospheric lines, or that these stars are binaries. Both suppositions are in contradiction with the fact that these lines are strongly blue-shifted. 
Table 3. Spectral types and absolute magnitudes of single WN stars in the SMC. The magnitudes are from Massey et al. (2003), although that of WR11 has been corrected as discussed in Foellmi et al. (2003a). WR12 appears as the hottest, and the second faintest WN star with hydrogen.

\begin{tabular}{lcc}
\hline \hline Star & Spectral Type & $M_{V}$ \\
\hline WR1 & WN3ha & -4.6 \\
WR2 & WN5ha & -5.2 \\
WR4 & WN6h & -6.2 \\
WR9 & WN3ha & -4.3 \\
WR10 & WN3ha & -3.6 \\
WR11 & WN4h:a & -4.7 \\
WR12 & WN3ha & -4.0 \\
\hline
\end{tabular}

If these absorption lines come from the presupposed "photosphere" of the WR star, it would be impossible to reach values as low as $50 \mathrm{~km} \mathrm{~s}^{-1}$ when the mean velocity of WR12 is about $160 \mathrm{~km} \mathrm{~s}^{-1}$ (assuming that the star has a systemic velocity similar to that of the SMC). This velocity difference is too large for a photosphere, and these lines must be formed in the wind itself.

As for binaries, they admit that the study by Foellmi et al. (2003a) "suggest that the binary fraction of WRs in the SMC is normal". This is half the truth. Foellmi et al. discussed the binary status of each of the WN stars in the SMC with not only the help of radial-velocities, but also line-profile variations, absolute magnitudes, spectral classification from a homogeneous set of spectra, archival photometric data and X-rays fluxes for most of the stars. Although the case of SMC-WR9 was not definitive, they have shown that SMC-WR1, WR2, WR4, WR10 and WR11 are certainly single stars, and they do show absorption lines in their spectra. WR12 is very similar to these WN stars in the SMC, especially WR1 and WR10.

We think that the presence of intrinsic hydrogen in the spectra of single WN stars in the SMC is inescapable. The H/He ratio (by number), obtained from a preliminary modelling of the spectra (Crowther 2000, private communication) even reaches values as high as 1 for WR9 and 2 for WR10. The spectral types of all the single WN stars in the SMC are reproduced in Table 3, along with the absolute magnitudes. It can be seen that WR12 fits in this subgroup of stars, especially with the faintest.

The presence of hydrogen has important implications for the formation of WR stars at low metallicity, and more precisely for the initial mass of their progenitors.

\subsection{The problem of the initial mass}

As in Massey et al. (2000), Massey et al. (2003) claimed that WR stars in the SMC come from only the highest mass (greater than $70 M_{\odot}$ ) stars, in accord with the expectations that at low metallicities only the most massive and luminous stars will have sufficient mass loss to become WR stars.

There is one important caveat in Massey et al. (2000) that led to wrong conclusions. They do not account for the influence of rotation (e.g. using the "old" evolutionary paths of
Schaller et al. 1992), although numerous pieces of evidence exist today that rotational effects modify strongly the evolution of massive stars, and therefore the shaping of a WR population at low metallicity. More precisely, rotating stars appear overluminous for their actual masses (Maeder \& Meynet 2000). Consequently, the estimation of the progenitor masses of WR stars in the Magellanic Clouds from cluster turn-offs are certainly too high, in addition to being in contradiction with the presence of hydrogen in faint single WN stars.

The problem with very high initial mass progenitors at low $Z$ is that they cannot explain the concomitant facts that WN stars in the SMC have a very early ionization subclass and hydrogen in their wind. These stars cannot have had high initial mass progenitors, since such masses would mean a high massloss during the Main-Sequence phase, and the hydrogen-rich envelope would have been removed by the time the WN phase was reached. If, because of the metallicity, the mass-loss is drastically reduced and some hydrogen is still left in the envelope, it is incompatible with the early ionization subclasses observed, because more massive WN stars are cooler, leading to a late spectral type.

On the other hand, relatively "low" mass (say 30-50 $M_{\odot}$ ) rotating progenitors are believed to produce early hydrogenrich WR stars at low metallicity.

\subsection{The question of rotation}

Rotation favours the formation of WR stars by two means: an increased internal mixing and an enhanced mass-loss rate during the MS phase (Maeder \& Meynet 2001). In the SMC, a mass-loss rate enhanced by rotation would be balanced by the low metallicity, while the enhanced internal mixing will drive hydrogen down to deeper layers, leading to WR stars that will remain "hydrogen-rich" longer. We can therefore expect theoretically similar values of the minimum mass of formation of a WR star, whatever the metallicity.

Moreover, in the context of the absence of rotation, the WR binary frequency was expected to be clearly metallicitydependent, since fewer single WR stars will be formed compared to those formed in binaries ${ }^{1}$. However, the binary frequency among WR stars has been shown to be similar, and statistically identical, both in the SMC (Foellmi et al. 2003a) and the LMC (Bartzakos et al. 2001; Foellmi et al. 2003b), and compatible with the galactic frequency, although this latter value is not reliable because of incompleteness. These similar values hide the opposite contribution of two different effects: at low $Z$, the mass-loss rate is lower, thus less angular momentum is lost during the Main Sequence, and the WR progenitors will have a larger mean rotation velocity (see e.g. Maeder \& Meynet 2000), favouring the formation of WR stars even for relatively low mass progenitors.

As for the early types observed in the SMC, Crowther (2000) has shown that for identical physical parameters (except the metallicity), wind modelling gives earlier subtypes at lower metallicity. If the rotation enhances the internal mixing,

\footnotetext{
1 We make the hypothesis that the influence of a companion on the formation of the WR component is independent of the metallicity.
} 
the increased abundance of helium in the atmosphere of the star reduces its opacity. The star will be more compact, bluer, and for a given mass it will be hotter. Since the temperature is directly related to the ionization subclass (e.g. Hamann et al. 1995), it explains the early types observed.

While the influence of rotation in short-period binaries is still hard to evaluate, it seems clear that at least the single WR stars in the SMC have been formed, or at least strongly influenced, by rotation.

\section{Conclusion}

We have obtained a set of 3 high- $S / N$ spectra spread over 10 days of the recently discovered WR star in the SMC. We have measured its $R V \mathrm{~s}$ and found them to be consistent with a constant velocity, indicating a probable single-star status. We have also measured the $R V \mathrm{~s}$ of hydrogen and helium absorption lines found in the spectra. Similar to what is seen in other SMC WN stars, the absorption lines have constant radial velocities and are strongly blue-shifted. Consequently, hydrogen must be present in the wind of the star, and the lines are formed along the line of sight through the relatively weak wind. Our spectral classification is WN3ha, while the evolutionary classification is eWNL.

The presence of hydrogen in single WN stars in the SMC raised the question of the mass of their progenitors, and the influence of metallicity and rotation on the formation of WR stars at low $Z$. We have shown that rotation effects on relatively "low" mass progenitors can better explain the whole set of properties of SMC-WR12 and other single, faint, hydrogenrich WN stars in the SMC.
Acknowledgements. C.F. is grateful to K. Aubel and O. R. Hainaut. C.F. also thanks L. Germany for a careful reading of the manuscript, and A. F. J. Moffat for valuable comments and for having brought to his attention the preprint of Massey et al. The author also acknowledges the referee (W.-R. Hamann) for comments and important improvements on the manuscript.

\section{References}

Bartzakos, P., Moffat, A. F. J., \& Niemela, V. S. 2001, MNRAS, 324, 18

Crowther, P. A. 2000, A\&A, 356, 191

Foellmi, C., Moffat, A. F. J., \& Guerrero, M. A. 2003a, MNRAS, 338, 360

Foellmi, C., Moffat, A. F. J., \& Guerrero, M. A. 2003b, MNRAS, 338, 1025

Hamann, W.-R., Duennebeil, G., Koesterke, L., Wessolowski, U., \& Schmutz, W. 1991, A\&A, 249, 443

Hamann, W.-R., Koesterke, L., \& Wessolowski, U. 1995, A\&A, 299, 151

Maeder, A., \& Meynet, G. 2000, A\&A, 361, 159

Maeder, A., \& Meynet, G. 2001, A\&A, 373, 555

Massey, P., \& Duffy, A. S. 2001, ApJ, 550, 713

Massey, P., Olsen, K. A. G., \& Parker, J. W. 2003, PASP, 115, 1265

Massey, P., Waterhouse, E., \& DeGioia-Eastwood, K. 2000, AJ, 119, 2214

Phelps, F. M. 1982, M.I.T. wavelength tables (M.I.T.)

Schaerer, D., \& Vacca, W. D. 1998, ApJ, 497, 618

Schaller, G., Schaerer, D., Meynet, G., \& Maeder, A. 1992, A\&AS, 96, 269

Smith, L. F., \& Maeder, A. 1998, A\&A, 334, 845

Smith, L. F., Shara, M. M., \& Moffat, A. F. J. 1996, MNRAS, 281, 163 